\title{
Innere Führung in Zeiten des Umbruchs: Zur Aktualität einer für obsolet erklärten Konzeption
}

\author{
Martin Kutz*
}

\begin{abstract}
Innere Führung, that is leadership development and civic education, is considered to be a particularity of the German Forces, the Bundeswehr. It manifests itself in various institutional forms. There are directives on Innere Führung, and in the legal provisions governing the military we find many principles which refer to Innere Führung. The paper is structured as follows: First: Do the new challenges require a reorientation? Secondly, the military patterns of reaction will be addressed. Third: Innere Führung in the context of rearmament during the East-West conflict. Fourth: Concept of Innere Führung. Finally a possible application of Baudissin's patterns of logic as »invented« by Count Baudissin in our times will be discussed.
\end{abstract}

Keywords: Konzeption Innere Führung, Professionalisierung, Logikstrukturen, Transformation

\section{Die neuen Herausforderungen - Zwang zur Umorientierung}

$\mathrm{D}$ ie Streitkräfte der europäischen Staaten stehen seit einigen Jahren vor z.T. völlig neuen Herausforderungen. Seit der große industrialisierte Krieg, der im Ost-WestKonflikt noch drohte, den so genannten neuen Kriegen das Feld räumen musste, spielt die Verteidigung der Nation keine Rolle mehr. Soldaten werden weit außerhalb des nationalen Territoriums mit Aufgaben betraut, die sie noch vor zwanzig Jahren weit von sich gewiesen hätten. Selbst dort, wo wie im jüngsten Irak-Krieg Streitkräfte noch mit traditionellen militärischen Kampfaufträgen eingesetzt werden, ist von einer Asymmetrie der Kontrahenten auszugehen und die Ausweitung der Kampfformen zu Partisanen- und Terror-Krieg mit ins Kalkül zu ziehen. Die neue Situation zwingt zum Nachdenken über vier Sachverhalte.

Zunächst gilt es, sich ein rational analysiertes Bild vom Krieg der Zukunft zu machen. Die neuen Herausforderungen asymmetrischer Kriegführung, von Bürgerkrieg, Bandenkrieg, Warlordism und Kriegsökonomie müssen in einem bisher nicht bedachten Ausmaß zum Maßstab militärischer Organisation, Ausbildungs- und operativer Konzepte gemacht werden.

Darum gilt es, die Legitimationsbasis für den Einsatz der Streitkräfte neu zu bedenken. Will man keine Söldnerarmeen haben, sondern den Staatsbürger in Uniform behalten, ist es sehr viel schwerer geworden, ihm einen Einsatz in Afrika, im Orient oder sonst wo in der Welt als notwendig einsichtig zu machen, zumal die Zivilbevölkerung sich immer weniger für ihre nationalen Streitkräfte interessiert und engagiert.

Damit ist das dritte Problemfeld angerissen. Sollen die Streitkräfte nicht politisch exterritorial werden, will man eine Kompatibilität von Streitkräften und nationaler Gesellschaft erhalten, so müssen Soldaten mit der Politik ihrer Nation, mit den politischen Zielen militärischer Einsätze wenigstens

* Dr. phil. Martin Kutz ist Wissenschaftlicher Direktor a.D. in Hamburg. Vorabdruck des Textes mit freundlicher Genehmigung des Wissenschaftlichen Forums für Internationale Sicherheit e.V. (WIFIS). Der Beitrag erscheint in Kürze in der Schriftenreihe von WIFIS in der Edition Temmen, Bremen. grundsätzlich vertraut und einverstanden sein. Eine in Europa noch weit verbreitete Auffassung, Soldaten hätten unpolitisch zu sein und müssten in jedem Falle fraglos jeden militärischen Auftrag ausführen, dürfte in Zukunft immer schwerer vermittelbar sein. Hier werden Armeen der zivilisierten Welt in Zukunft Probleme haben.

Das führt zum vierten Punkt. In den demokratischen Gesellschaften Europas wird sich auf Grund der Gesellschaftsentwicklung ein immer kleinerer Teil junger Menschen für die militärische Laufbahn entscheiden, weil auch militärische Traditionen und Verhaltensmuster im Dienstalltag sich mit den Vorstellungen von einem interessanten und befriedigenden Berufsalltag der heranwachsenden Generation nicht mehr vereinbaren lassen. Bleibt man beim traditionellen militärischen Stil, wird das gesellschaftliche Rekrutierungssegment immer kleiner und politisch enger. Die Kompatibilität mit Gesellschaft und Gesellschaftsentwicklung wird so schleichend aber langfristig zwingend zerstört.

All diese Probleme haben europäische Streitkräfte schon heute oder müssen davon ausgehen, dass sie ihnen in Zukunft nicht ausweichen können.

\section{Die militärischen Reaktionsmuster}

Erkennbar sind derzeit zwei Reaktionen auf diese Problemlage (vgl. Schema »Denkwege des militärischen Professionalismus«).

Die erste und am meisten verbreitete ist, auf die technische Seite der Herausforderung mit technokratischen Mitteln zu reagieren. Es werden neue Taktiken entwickelt, Ausbildung und Ausrüstung angepasst. Die Ursachen und Weiterungen aber werden weitgehend ausgeblendet. Hier dominiert ein konservativer Traditionalismus, dessen Ausgangspunkt des Denkens traditionale Berufsbilder sind. Diese werden historisch legitimiert und sind im Bewusstsein dieser Vertreter in den Soldatentugenden manifestiert. Sie (allein) garantieren dann die Kriegstüchtigkeit. Zu Disziplinfragen wird auf traditionelle militärische Verhaltensmuster verstärkt zurückgegriffen. Da 


\section{Denkwege des militärischen Professionalismus}

Progressiv-rational

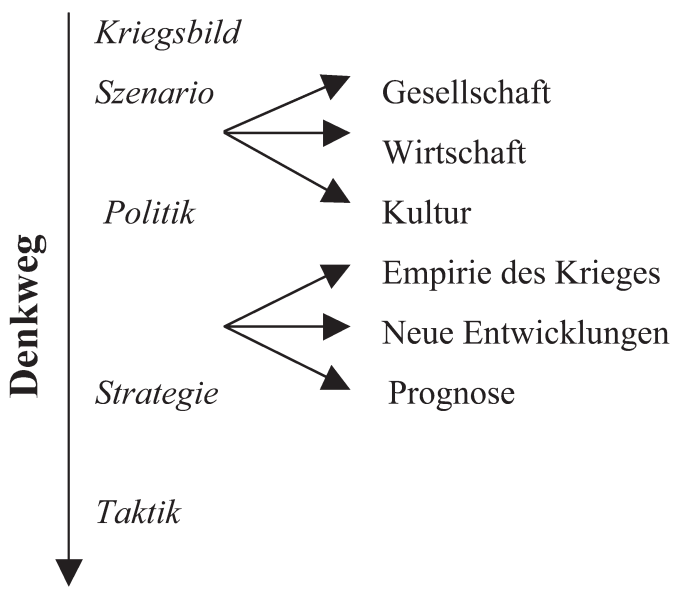

Konservativ-traditional

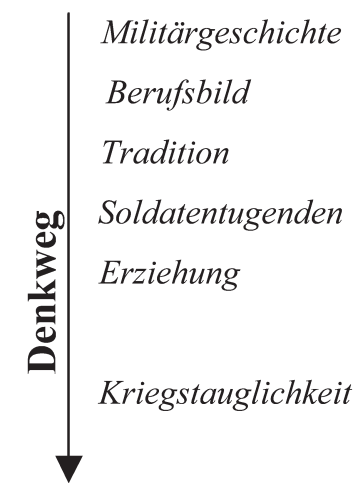

das aber im Einsatz selber nicht mehr ohne Weiteres funktioniert, wird die Diskrepanz zwischen militärischer Norm und militärischer Praxis größer.

Die zweite Reaktion ist bisher in einem kleinen Kreis militärischer Planer und Analysten erkennbar. Sie diskutieren die Probleme zur Zeit noch theoretisch - was dringend geboten ist - und versuchen, in ihrem Umfeld die Sensibilität für ihre Gedanken und Vorstellungen zu wecken. Sie gehen von einem modernen Bild des zukünftig zu erwartenden Krieges aus, leiten daraus konkrete Szenarien ab, berücksichtigen bei der politischen Entscheidung Gesellschaftsentwicklung, Wirtschaft und kulturelle Faktoren und leiten daraus erst Strategie und Taktik ab. In den deutschen Streitkräften hatten solche Ideen in den 80er und 90er Jahren des 20. Jahrhunderts nur geringe Resonanz. Probleme wurden so verschleppt und Veränderungen verhindert.

In Deutschland hat letzteres jüngst eher aus politischer Tradition als aus theoretischer Einsicht die Diskussion um die Innere Führung wieder belebt. Damit ist hier an Vorstellungen aus den 50er Jahren des 20. Jahrhunderts angeknüpft, die alle aufgeführten Probleme in einem anderen militärstrategischen, gesellschaftlichen und politischen Kontext schon einmal stringent durchexerziert haben. Zwar war die Wirkung dieser Konzeption Innere Führung begrenzt, weil der politische und vor allem militärische Traditionalismus vehement dagegen opponierte. Aber wesentliche Aspekte des Konzeptes haben sich dann in den 70er Jahren doch in den deutschen Streitkräften durchgesetzt, sehr zum Nutzen von Armee und Gesellschaft. Diesen Prozess der langsamen und teilweisen Durchsetzung der Grundzüge der Inneren Führung hier darzustellen, würde zu weit führen. Wichtig ist aber zweierlei.

Erstens, dass das Konzept aus allgemeinen Prinzipien auf eine spezielle politische, gesellschaftliche und militärstrategische Situation zugeschnitten wurde. Die Folge davon ist, dass mit dem Konzept die Vorstellung verbunden wird, es sei historisch möglicherweise überholt und zudem nur für die speziellen deutschen Verhältnisse brauchbar. Diese Sicht verkennt, dass Grundlage für die konkrete historische Ausformulierung stringente logische Analyseschemata sind, die vom historischen Kontext unabhängig auch in der Gegenwart gelten.

Zweitens, dass auch in der Bundeswehr die Konzeption der Inneren Führung in ihrer vollen Tragweite nicht überall verstanden ist, dass sie sogar von Traditionalisten abgelehnt wird, und dass sie über lange Zeit, besonders seit den 80er Jahren auf eine manipulative Motivationstechnologie reduziert wurde.

Es ist demnach notwendig, die Konzeption Innere Führung in ihrer logischen Stringenz zu verdeutlichen und die Verbiegungen und Missverständnisse der militärischen Alltagspraxis unberücksichtigt zu lassen.

\section{Innere Führung im Kontext von Wiederbe- waffnung und Ost-West-Konflikt}

Der »Erfinder « der Inneren Führung ist Wolf Graf von Baudissin², Generalstabsoffizier bei Rommel in Nordafrika bis zu seiner Gefangennahme 1941 bei Tobruk. Erste Überlegungen in der Gefangenschaft lassen Grundzüge der späteren Konzeption erkennen. Ausgangspunkt seiner Überlegungen damals ist seine moralisch-ethisch begründete Opposition zum Hitlerregime.

In dieser Opposition sieht er sich nach dem Kriege voll gerechtfertigt, als er den vollen Umfang der politischen und militärischen Verbrechen des Regimes erfährt. Für ihn ist der Weg in dieses Verbrechensregime die Konsequenz einer historischen Fehlentwicklung des Militärs und des Verhältnisses

2 Von seinen zahlreichen Denkschriften, Reden, Aufsätzen und Stabsstudien sind wesentliche Teile in folgenden Veröffentlichungen zu finden: Wolf Gra von Baudissin, Soldat für den Frieden. Entwürfe für eine zeitgemäße Bundeswehr, München 1969, ders., Nie wieder Sieg! Programmatische Schriften 1951 - 1981, München 1982; ders. und Dagmar Gräfin zu Dohna, ...als wären wir nie getrennt gewesen. Briefe 1941-1947, herausgegeben mit einer Einführung von Elfriede Knoke, Bonn 2001. 
von Militär und Politik und Militär und Gesellschaft. Für ihn gibt es deshalb in der deutschen Militärgeschichte nur zwei legitime Anknüpfungspunkte für die neuen Streitkräfte. Der erste sind die preußischen Reformen von 1808/18, so wie sie Scharnhorst und Gneisenau damals konzipiert hatten, und nicht die verstümmelten Formen, wie sie dann tatsächlich Platz griffen. Der zweite Anknüpfungspunkt ist für ihn der Widerstand gegen Hitler, der sich für ihn im Putsch vom 20. Juli 1944 symbolisierte. Bei diesem Putsch waren viele Freunde Baudissins aktiv und haben den Umsturzversuch mit dem Leben bezahlt. Beide historische Bezugspunkte zeigen, dass Baudissin ein konservativer Mensch war, konnte er sich doch offensichtlich politische Legitimation für eigenes Handeln nur als historisch legitimiertes Handeln vorstellen.

1951 tritt er in die deutsche Planungsbehörde für die Wiederbewaffnung ein. Als Bedingung für seinen Eintritt verlangt er, die gescheiterten Teile der preußischen Reformen von 1808 bis 1818 zur Grundlage seiner Arbeit machen zu dürfen. ${ }^{3}$ Diese Teile, die ihn interessieren, betreffen den »Bürgersoldaten «. Die ethisch-moralischen und politischen Prinzipien von 1808 überträgt er auf die Situation von 1951/61 in allen seinen Arbeiten. Konkret heißt das, dass er ein ethisch fundiertes Konzept einer demokratieverträglichen Armee für die strategische Situation des Ost-West-Konfliktes unter der Drohung des Atomkrieges entwirft. Dieses ist allerdings in seinen Studien eher versteckt als offen erkennbar, denn Baudissin hat in einem vernetzten Mehrebenendenken nur praktische Probleme bearbeitet. Es gibt bei ihm keinen Text, mit Ausnahme eines Aufsatzes über die Konsequenzen des Atomkrieges, der seine theoretischen Grundlagen systematisch darstellen würde. Die Systematik muss aus Texten zu verschiedensten Themen herausgefiltert werden, und sie zeigt sich dann als logisches Denksystem, das an Carl von Clausewitz' Buch vom Kriege geschult ist. ${ }^{4}$

\section{Die Konzeption der Inneren Führung}

Baudissin geht von drei Prämissen des Denkens aus. Die erste ist, dass Militär, Militärpolitik und Strategie von einem modernen, zukunftsoffenen Bild vom zukünftigen Krieg aus gedacht werden müssen. Er ist schon in den fünfziger Jahren einer der wenigen Militärs, der die Epochenbedeutung der Atomwaffen verstanden hat, sie zu politischen, strategischen Waffen erklärt und daraus Kriegsverhinderung durch militärische Abschreckung als zwingende Notwendigkeit ableitet. Der Soldat ist also kein Soldat für das Kriegführen, sondern seine erste Aufgabe ist der Erhalt des Friedens (Soldat für den Frieden). Aus der strategischen Situation leitet er den Zwang zur Bündnisorientierung nach Westeuropa und zu den USA $\mathrm{ab}$, dazu den Zwang zur politischen Kooperation auch mit dem militärischen Gegner und in späteren Jahren ein Kon-

3 Martin Kutz, Reform als Weg aus der Katastrophe. Über den Vorbildcharakter der Preußischen Reformen 1808-1818 und die Vergleichbarkeit der Situationen von 1806 und 1945 für Arbeit und Denken Baudissins, in: H. Linnenkamp, D.S. Lutz (Hg.), Innere Führung. Zum Gedenken an Wolf Graf von Baudissin, Baden-Baden 1995.

4 Dazu Martin Kutz, Historische Wurzeln und historische Funktion des Konzeptes Innere Führung (1951-1961), in: K. Kister, P. Klein (Hg.), Staatsbürger in Uniform - Wunschbild oder gelebte Realität?, Baden-Baden 1989. zept zur »kooperativen Rüstungssteuerung «. Aus der atomaren Drohung leitet er ab, dass Soldaten extrem selbständig auf dem Schlachtfeld agieren und deshalb intrinsisch motiviert sein müssen. Auch die traditionellen Befehls- und Gehorsamsverhältnisse können so nicht mehr tragen. Sie müssen durch einen kooperativen Führungsstil abgelöst werden.

Die ethische Fundierung des Soldatenberufes ist bei Baudissin christlich-protestantisch und zugleich philosophisch begründet. Hier ist seine Bindung an Menschenrechte und Bürgerrechte letztlich verankert. Dies überträgt er auf die Verfassungsartikel des Grundgesetzes der Bundesrepublik und leitet daraus wiederum ab, das die oberste Verpflichtung des Soldaten der Erhalt des Friedens ist, somit Kriegsverhinderung notwendig und zur Sicherung des Friedens die politische Kooperation unumgänglich. Das erzwingt aber auch für die militärische Alltagspraxis die Einhaltung demokratischer Verhaltensmuster, kurz die Bindung an die Prinzipien der Inneren Führung.

Dritte Prämisse seines Denkens ist die demokratische Gesellschaft. Das heißt mehr, als der Bezug zum demokratischen Staat (wenigstens in den 50er Jahren des 20. Jahrhunderts in Deutschland). Es geht ihm um eine demokratische, im Alltag praktizierte Lebensform, die er auch fürs Militär einfordert. Das tut er in einer Zeit, als die deutsche Gesellschaft selber noch in großer Distanz zur Demokratie lebt. Politisch leitet er davon die zivile Kontrolle des Militärs ab, die Sicherung der Menschen- und Bürgerrechte im Militär selber und die Feststellung, dass der Soldatenberuf ein politischer Beruf ist, ein Beruf, der ohne den Bezug zur Politik sinnlos wird und Gefahr läuft, zum Soldknecht zu werden. Die folgende Matrix soll diese Formalstruktur der logischen Bezüge verdeutlichen.

\section{Matrix logischer Bezüge im Denken Baudissins}

Diese Matrix lässt auch erkennen, dass es im Prinzip gleichgültig ist, auf welcher Ebene ein Problem lokalisiert ist. Es ist immer auch erkennbar, wie es in den Gesamtzusammenhang aller Probleme militärischer Sicherheit eingebunden ist. In diesem Schema denken heißt dann auch, Probleme nicht isoliert wahrzunehmen, sondern zugleich auch alle Dependenzen, mit denen sie verknüpft sind. Konsequenz aller Ableitungen in diesem Schema ist aber auch, dass im Militär selber Innere Führung immer gefordert ist.

Der Begriff Innere Führung ist in gewisser Weise irreführend. Ursprünglich wurde vom Inneren Gefüge gesprochen. Die Gegner Baudissins, seine Offizierskameraden aus der Wehrmacht, die sich eine Demokratisierung der Armee nicht vorstellen konnten und wollten, hatten das Wort in Inneres Gewürge umgetauft, um Baudissins Vorstellungen lächerlich zu machen. Das Wort Innere Führung wurde deshalb als Ersatzbegriff erfunden. ${ }^{5}$

Der ursprüngliche Begriff ist zutreffender, macht er doch deutlich, dass es nicht nur um eine Führungsphilosophie ging und geht, sondern die militärische Struktur und die Verhaltens-

5 Eine detaillierte Schilderung der Auseinandersetzungen findet sich bei Dietrich Genschel, Wehrreform und Reaktion. Die Vorbereitung der Inneren Führung 1951-1956, Hamburg 1972. 


\begin{tabular}{|c|c|c|c|}
\hline Hand- & Kriegsbild & Ethisches Fundament & Demokratische Gesellschaft \\
\hline Politik & $\begin{array}{ll}\text { - } & \text { West- und } \\
\text { Bündnisorientierung } \\
\text { - } & \text { Politische } \\
\text { Kooperation } \\
\text { - } \quad \text { Kooperative } \\
\text { Rüstungssteuerung }\end{array}$ & $\begin{array}{ll}\text { - } & \text { Friedenssicherung } \\
\text { - } & \text { Politische } \\
\text { Kooperation }\end{array}$ & $\begin{array}{l}\text { - } \text { Demokratische zivile } \\
\text { Kontrolle des Militärs } \\
\text { - Sicherung der Grundrechte } \\
\text { im Militär } \\
\text { - Soldatenberuf ein politischer } \\
\text { Beruf }\end{array}$ \\
\hline Strategie & $\begin{array}{ll}\text { - } & \text { Kriegsverhinderung } \\
\text { - } & \text { Abschreckungs- } \\
& \text { strategie }\end{array}$ & - $\quad$ Kriegsverhinderung & $\begin{array}{ll}\text { - } & \text { Kriegsverhinderung } \\
\text { - } & \text { Abschreckungs-strategie }\end{array}$ \\
\hline Militär & $\begin{array}{ll}\text { - } & \text { Soldat für den Frieden } \\
\text { - } & \text { Innere Führung }\end{array}$ & $\begin{array}{ll}\text { - } & \text { Soldat für den } \\
\text { Frieden } \\
\text { - } \quad \text { Innere Führung }\end{array}$ & $\begin{array}{ll}\text { - } & \text { Demokratiekonformes } \\
\text { - } & \text { Militär } \\
\text { Integration von Militär in der } & \text { demokratischen Gesellschaft } \\
\text { - } & \text { Staatsbürger in Uniform } \\
\text { (Bürgersoldat) } \\
\text { - }\end{array}$ \\
\hline
\end{tabular}

prinzipien mit umschließt. Da Baudissin seine individuelle ethische Orientierung in den Menschen- und Bürgerrechten der deutschen Verfassung allgemein verbindlich formuliert sieht, kann er formal aus der Analyse eines zutreffenden Kriegsbildes und durch die Ableitungen aus den Prinzipien einer demokratischen Gesellschaft Innere Führung als logische Konsequenz rationalen Denkens formulieren (vgl. Schema »Zusammenhängende Schlüsselbegriffe Baudissins«). Die folgende Zusammenstellung der Schlüsselbegriffe zeigt ihre Bezüge und die Logikstruktur der Konstruktion Innere Führung.

\section{Zusammenhängende Schlüsselbegriffe Baudissins}

Aus dem Kriegsbild seiner Zeit, einer umfassenden Analyse aller damaligen erkennbaren Kriegsformen, die aber alle von der Drohung des Atomkrieges überschattet sind, leitet er unter Beachtung der neuesten technologischen Trends ab, dass moderne Streitkräfte technologieorientierte voll mechanisierte Organisationen sind. Die Atomwaffen als politische Waffen und die Situation des Ost-West-Konfliktes erzwingen die politische Zuverlässigkeit des Militärs als Voraussetzung ihrer Funktionstüchtigkeit. Wegen der die Menschheit gefährdenden Vernichtungskraft der Atomwaffen ist Kriegsverhinderung allererste Aufgabe von Militär, und diese unter den gegebenen Umständen nur durch militärische Abschreckung möglich.
Prinzipien einer demokratischen Gesellschaft sind die individuellen Freiheitsrechte, also Menschenrechte und Menschenwürde. Sie konkretisieren sich politisch und organisatorisch im Rechtsstaat. Auf die militärische Organisation und den einzelnen Soldaten herunter gebrochen heißt das Innere Führung.

Ihre Prinzipien sind die Vorstellung vom Soldaten als Staatsbürger in Uniform, der seine Freiheitsrechte eben nicht am Kasernentor aufgeben muss und auch das Recht auf politische Betätigung als Soldat behält. Insbesondere für den militärischen Vorgesetzten, der vor 50 Jahren eher ein Mussdemokrat denn einer aus eigener Überzeugung war, war demokratische Orientierung nur in einem Bildungsprozess zu erwarten. Baudissins Vorstellung von Bildung schloss traditionelle bürgerliche Bildungsinhalte ebenso ein wie die vehement geforderte politische Bildung. Sie sollte zu demokratieverträglichem Verhalten führen und so dem Rekruten wie jedem anderen Soldaten die Erfahrung vermitteln, dass die Freiheit, die er gegen die totalitäre Bedrohung verteidigen sollte, im militärischen Alltag genauso gelte wie im Zivilleben. Diese Erfahrung sollte auch Indifferente zu Demokraten »erziehen «. Erreichbar in der Praxis aber ist dieses Ziel nur, wenn ein kooperativer Führungsstil die individuellen Fähigkeiten und Orientierungen der Geführten berücksichtigt. Eine an den technischen Erfordernissen der Moderne orientierte Disziplin muss dann auch 


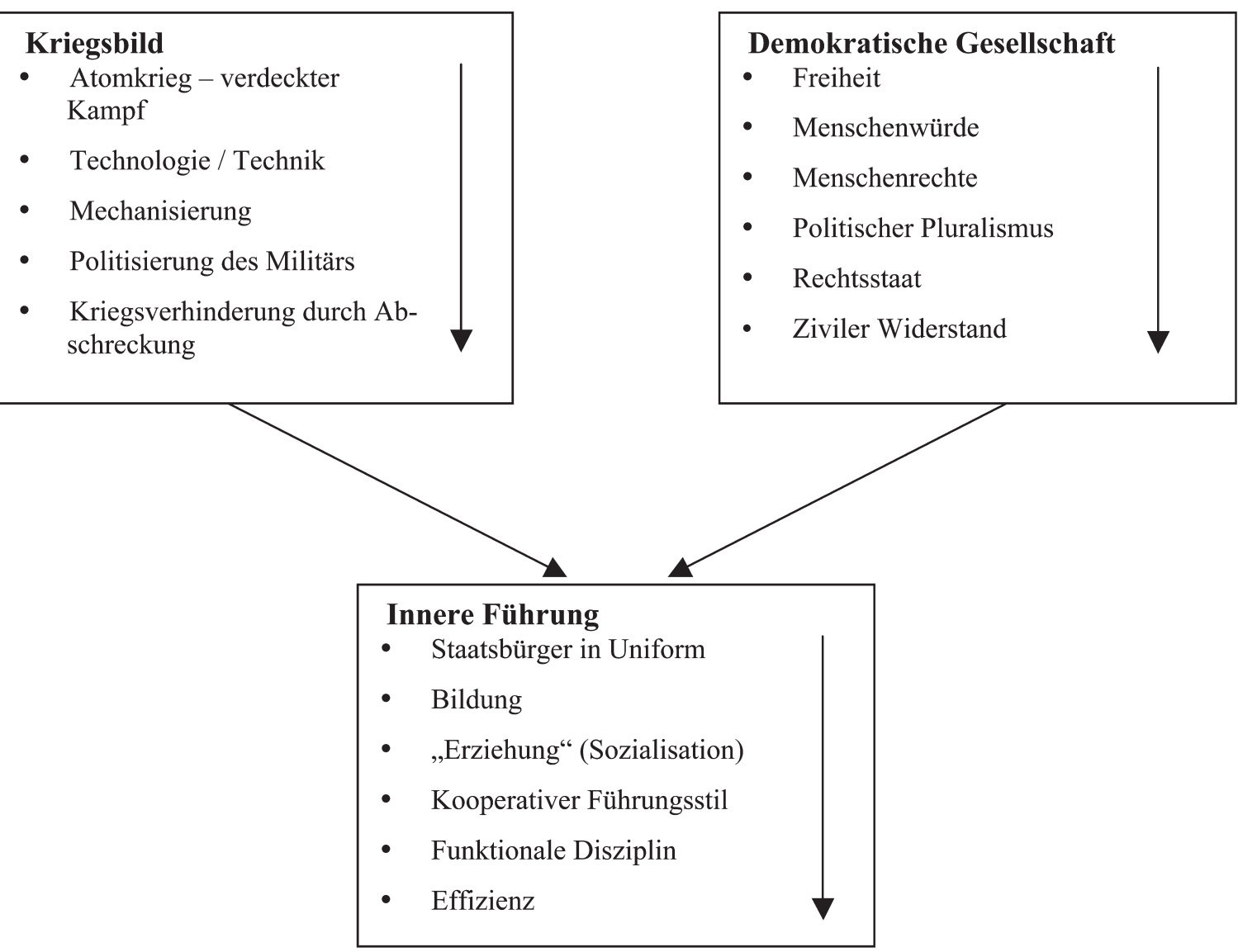

die traditionale Kasernenhofdisziplin ablösen. Erst wenn alle diese Aspekte erfüllt sind, ist nach dieser Vorstellung die Effizienz der Streitkräfte gegeben.

Notwendig ist also, dass sich moderne Streitkräfte am Zukunftskrieg und nicht an der Tradition orientieren, dass sie in die demokratische Landschaft eingebettet sind und der einzelne Soldat seine demokratischen Erfahrungen, Einstellungen und Rechte auch im militärischen Alltag berücksichtigt findet. Der folgende Überblick über die Baudissinschen Logikstrukturen soll den Gesamtzusammenhang des Denksystems von Baudissin verdeutlichen. Die aufgelisteten Begriffe betreffen die strategische Situation im Ost-West-Konflikt der 50er und 60er Jahre des letzten Jahrhunderts.

\section{Logikstrukturen von Begriffsclustern bei Baudissin}

Man kann dieses Schema in zwei Richtungen lesen. Nimmt man die Waagerechte, so wird die politisch-strategische Ebene sichtbar. Aus dem Kriegsbild wird, wie schon erläutert, das Friedensgebot fürs Militär abgeleitet. Der zweite Ausgangspunkt, die Demokratie, führt in Verbindung mit dem Kriegsbild ebenfalls zum Friedensgebot und aus beiden, Kriegsbild und demokratischer Ordnung wird das demokratieverträgliche Militär abgeleitet. Dieses demokratieverträgliche Militär braucht, ja erzwingt aus der Logik der Sache den Staatsbürger in Uniform, den Bürgersoldaten. Kriegsbild, Demokratie und demokratieverträgliches Militär bedingen sich also gegenseitig. Die zweite Ebene des Schemas beschreibt die Konsequenzen für das Militär, die Organisationsebene also. Die Konsequenzen aus den damals modernen militärischen Strukturen sind hier abgebildet. Dabei wird eine zweite logische Bezugsebene deutlich, die sich in der Vertikalen zeigt.

Das moderne Kriegsbild hatte zur Folge, dass die Streitkräfte technologieorientiert und voll mechanisiert waren. Ihre Aufgabe der Kriegsverhinderung konnte aber nur tatsächlich erfüllt werden, wenn sie auch politisch zuverlässig waren. Die Bürgerkriegssituation in Deutschland mit zwei Armeen in den unterschiedlichen Machtblöcken und die ideologische Auseinandersetzung mit dem Kommunismus, die in Deutschland auf Grund der Teilung des Landes besonders stark war, nötigte zu dieser Vorstellung. Daraus leitet sich bei Baudissin ein neuer Effizienzbegriff ab. Effizient sind nur Streitkräfte, die in sich militärisch funktionale Aufgaben und politische Zuverlässigkeit vereinen. Der Soldatenberuf ist danach ein politischer Beruf.

Aus der gleichen Technologieorientierung wird unter Hinzufügung des neuen Effizienzbegriffes und der Berücksichtigung von Menschenwürde und Rechtsstaatlichkeit auch eine neue Disziplin abgeleitet. Sie wird funktional definiert, von den technischen und politischen Erfordernissen des Militärs abgeleitet. Die traditionelle Kasernenhofdisziplin, die in Deutschland im vordemokratischen Militär auch zur Erzwingung fraglosen Gehorsams durchexerziert wurde, wird strikt abgelehnt.

Die dritte Ableitung aus der Technologieorientierung wird durch die Hinzufügung der individuellen Freiheitsrechte des Soldaten vorgenommen. Daraus ergibt sich, dass der fraglose 
- Kriegsbild

- Atomkrieg

- Abschreckung

- Soldat für den Frieden
- Demokratie

- Kriegsverhinderung

- Staatsbürger in Uniform

- Erziehung als demokratische Sozialisation

- Soldat für den Frieden
- Demokratieverträgliches Militär

- $\quad$ Bildung

- Erziehung als demokratische Sozialisation

- Demokratische Traditionen

- $\quad$ Staatsbürger in Uniform

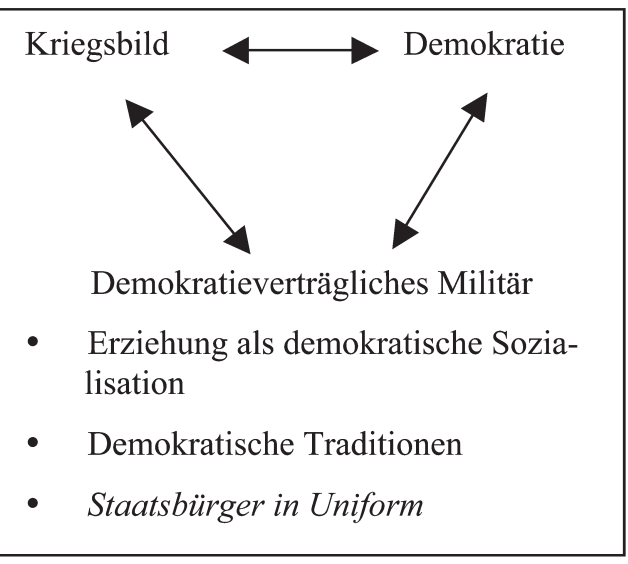

- Technologieorientierung

- Mechanisierung

- Kriegsverhinderung

- Demokratische Zuverlässigkeit

- Effizienz
- Technologieorientierung

- Mechanisierung

- $\quad$ Effizienz

- Menschenwürde

- Rechtsstaat

- Disziplin

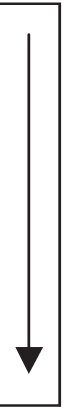

- Technologieorientierung

- Mechanisierung

- Freiheit

- Menschenwürde

- Kooperativer Führungsstil

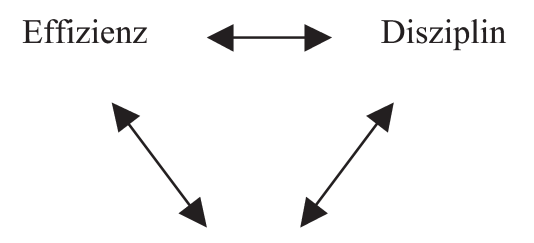

Kooperativer Führungsstil
Gehorsam und damit der autoritäre Führungsstil vergangener Zeiten durch kooperative Verhaltensmuster abgelöst werden muss. Zwei Gründe sind also maßgeblich. Erstens, dass die demokratische Ordnung das Alte verbietet, zweitens aber, dass die moderne Technik so komplex ist, dass ein autoritärer Führungsstil ihre optimale Nutzung verhindern würde. Hauptgrund ist, dass der militärische Führer gar nicht mehr in der Lage ist, alle technischen Funktionen korrekt zu bedienen und somit auch seine Untergebenen nicht mehr kontrollieren kann. Er ist auf Kooperation und guten Willen seiner Soldaten angewiesen.
Somit ergibt sich auf der Organisationsebene von Militär ein funktionaler und logischer Zusammenhang von einer neuen Effizienzvorstellung, einer neuen Auffassung von Disziplin und einem ebenso neuen militärischen Führungsstil.

\section{Die Anwendung der Logikmuster Baudissins auf die Gegenwart: Erste Überlegungen}

Innere Führung ist mehr als eine Motivationstechnologie für Soldaten und die Denkmuster, die logische Struktur dieses 
Denkens, sind geeignet, auch die Probleme der Gegenwart adäquat zu beschreiben und logisch korrekte Schlüsse für die militärische Praxis daraus zu ziehen. ${ }^{6}$ Dies soll nun mit Hilfe der folgenden Übersicht versucht werden.

\section{Aktuelle Probleme in den Logikstrukturen Baudissins}

Ausgangspunkte für die Analyse sind, wie bei Baudissin in der Vergangenheit, Kriegsbild, demokratische Gesellschaft und demokratischer Staat. Darunter sind stichwortartig die neu zu bedenkenden Faktoren aufgeführt. Jeder dieser drei Ausgangspunkte hat für das Militär logisch notwendige Konsequenzen. Sie betreffen die militärischen Strukturen, die Veränderungen beim Personal, weil Soldaten von heute andere Sozialisationstypen darstellen als vor 50 oder 100 Jahren, und die Erfordernisse einer neuen Legitimation von Militär und militärischen Einsätzen.

Wenn diese Überlegungen wirklich in die Praxis umgesetzt werden, werden die Streitkräfte in Zukunft anders aussehen. Sie werden eine neue Struktur haben, ihr Führungspersonal aller Ebenen der Hierarchie wird ein neues kooperatives, demokratisches Führungsverhalten lernen und man wird sich der Mühe unterziehen, dem Soldaten den politischen Sinn und Zweck von Militär und von den jeweiligen Einsätzen zu erklären.
Über eine Tatsache als Konsequenz dieser Entwicklung muss sich die politische Führung wie das Militär im Klaren sein: Man wird nicht mehr aus einer politischen Interessenabwägung diese Soldaten beliebig in den Krieg schicken können, es sei denn, man hat keine Hemmungen, sich auf professionelle Söldnertruppen einzulassen.

\subsection{Innere Führung und Transformation}

Die Auslandseinsätze der Bundeswehr werfen lange Schatten. Die tradierten Vorstellungen vom Soldatenberuf aber werfen die tiefsten Schatten. In Krisenzeiten, also immer, wenn es unter veränderten Bedingungen schwierig wird, wird die Flucht in die Geschichte und die Tradition angetreten. Ein unreflektierter Teil dieser Tradition ist die Vorstellung vom Soldaten als einem unpolitischen Gewalttechnokraten, oder, wie es der Inspekteur des Heeres formuliert hat, die vom »atavistischen High-Tech-Kämpfer«. Das Faszinierende an dieser Flucht ist, dass sie umso lauter betrieben wird, je weniger sie in der Realität eine Entsprechung findet. Denn noch kein Soldat der Bundeswehr ist im Kampf getötet worden, seit die neuerliche Rede vom Soldatischen und vom Kämpfer grassiert.

Dieser Rückzug aufs Historische, auf Tradition und Kämpfertum wird verknüpft mit einem offenen oder versteckten Angriff auf die Innere Führung. Sie ist entweder nicht mehr

\begin{tabular}{|c|c|c|}
\hline Kriegsbild & Gesellschaft & Politik \\
\hline ethnisch-religiöse Bürgerkriege & postindustrielle Gesellschaft & Parteiendemokratie \\
\hline Terrorismus & Globalisierung & Mediendemokratie \\
\hline Warlordism & Individualisierung & Entnationalisierung \\
\hline „Neue Kriege“ & Mulitkulturalismus & Ökonomisierung \\
\hline Massenvernichtungswaffen & Milieuorientierung & politische Fragmentierung \\
\hline $\begin{array}{l}\text { Militärstrukturen } \\
\text { weg von der Massenarmee } \\
\text { weg von schweren Waffen } \\
\text { hohe Beweglichkeit } \\
\text { extreme Logistikabhängigkeit } \\
\text { High-tech-Ausrüstung } \\
\text { electronic warfare }\end{array}$ & \begin{tabular}{|l} 
Veränderung des Rekrutierungspotentials \\
- neuer Menschentyp \\
- Gefahr der Segmentverengung \\
neue Anforderungen an Lernfähigkeit \\
- und Flexibilität \\
extreme Spezialisierung
\end{tabular} & $\begin{array}{l}\text { politische Legitimation militärischer } \\
\text { Aktionen } \\
\text { - Militär unter Aufsicht der Medien } \\
\text { - Zustimmung der Gesellschaft } \\
\text { - } \quad \text { politische Begründung auch für } \\
\quad \text { Soldaten }\end{array}$ \\
\hline $\begin{array}{l}\text { Ergebnis: } \\
\text { Neue militärische Strukturen }\end{array}$ & $\begin{array}{l}\text { Ergebnis: } \\
\text { Neues Führungsverhalten }\end{array}$ & $\begin{array}{l}\text { Ergebnis: } \\
\text { politische Bildung für Soldaten }\end{array}$ \\
\hline
\end{tabular}

6 Einen Versuch, Geschichte und Zukunft der Inneren Führung im Sinne Baudissins neu zu interpretieren findet man bei Martin Kutz (Hg.), Gesellschaft, Militär, Krieg und Frieden im Denken von Wolf Graf von Baudissin. BadenBaden 2004. In diesem Band haben dreizehn Autoren unter Beteiligung des Generalinspekteurs der Bundeswehr sich dieser Interpretationsaufgabe gestellt. 
»zeitgemäß« oder sie ist eine »Friedens- und Kasernenhoftheorie«, die für den Einsatz nicht taugt. Bestenfalls muss sie »auf den Prüfstand « oder »weiterentwickelt« werden. Innere Führung offen abzulehnen hat keiner den Mut, da dies als politisch nicht durchsetzbar bewertet wird. Manche wären sie jedoch gerne auf stille Weise los. Gelänge dies, wäre man auch das »Politische los. Den Primat der Politik garantierten dann die Generale. Ihre Untergebenen müssten dann nur noch gehorchen. Dieser gehorsame Subalterne wäre dann auch die politische Verantwortung für sein Tun los. Die Bundeswehr könnte dann in eine Spezialfirma für legitimes Gewaltmanagement umgeformt werden. Die Vorbilder dafür gibt es im Ausland. Nur fällt auf, dass diese meistens ihre Aufgabe nur schlecht erfüllen. Dort, wo einigermaßen funktioniert, was Militär leisten soll, ist erstaunlicherweise viel Innere Führung im Spiel.

Im Augenblick gibt es zwei Hauptaufgaben für die Bundeswehr, die eng miteinander verkoppelt sind. Die erste besteht darin, aus der Bundeswehr wieder ein zukunftstaugliches Instrument zu formen und die zweite in ihren Auslandseinsätzen, die den Zwang zur Umstrukturierung der Streitkräfte ausgelöst und aufrechterhalten haben. Auffällig ist, dass in beiden Feldern Innere Führung praktiziert wird und nicht ersichtlich ist, dass es ohne sie überhaupt möglich wäre, die gewünschten Leistungen zu erbringen.

Schon seit den 1970er Jahren kann man beobachten, dass der hauptsächliche Impuls, Innere Führung zu praktizieren, aus den gesellschaftlichen und individuellen Erfahrungen der Soldaten erwächst und nicht auf Wissen, Überlegung und militärischer Ausbildung beruht. Man verhält sich trotz aller militärisch-folkloristischer Tradition als Demokrat. Das erklärt auch, warum sich diejenigen, die sich zur Inneren Führung distanziert äußern oder in Teilbereichen auch distanziert verhalten, Innere Führung in vielen Aspekten trotzdem praktizieren.

Nun sind die Veränderungsprozesse in der Bundeswehr die gravierendsten seit Mitte der 70er Jahre des zwanzigsten Jahrhunderts. Was mit dem Stichwort »Transformation « angestrebt und beschrieben wird, wird von vielen Soldaten als ein nur technokratischer Prozess verstanden. Gegenstand der Überlegungen sind deshalb in erster Linie Organisationsfragen, also wie viel von welcher Waffengattung in welcher organisatorischen Form gebraucht wird. Dazu kommen die Überlegungen zur Umrüstung und praktischen Ausbildung, die auf die neuen Aufgaben zugeschnitten werden müssen. Und als wichtigste Einschränkung der Handlungsmöglichkeiten wird die Haushaltslage bezeichnet.

Wenn man aber die interne Diskussion genauer beobachtet, zeigen sich massive Defizite in der realitätsgerechten Wahrnehmung der neuen Situation. Das hat ganz wesentlich mit der technokratischen Grundorientierung im Offizierkorps zu tun, was heißt, dass wesentliche Zusammenhänge wie sie das Konzept der Inneren Führung in seiner ursprünglichen Form korrekt analysieren half, heute nicht oder zumindest nicht hinreichend verarbeitet werden:

1. Bis heute gibt es kein konsistentes und zugleich anerkanntes Bild vom zukünftigen Krieg. Darüber systematisch nachzuden- ken ist tabuisiert. Das Beste, was bisher dazu in der Bundeswehr produziert wurde, ist die Studie über die Streitkräftefähigkeiten im einundzwanzigsten Jahrhundert vom Zentrum für Analysen und Studien der Bundeswehr ${ }^{7}$, auch wenn die Studie nicht in allen Aspekten den Erfordernissen gerecht wird. Es ist das erste Mal in der Geschichte der Armee, dass so etwas überhaupt geleistet wurde.

2. Konsequenz eines solchen Defizits und der Tabuisierung des Nachdenkens über zukünftige Kriege auf politischer und militärischer Ebene ist, dass als natürliche Folge auch keine schlüssige Strategie erkennbar ist. Damit wird das nächste Defizit produziert.

3. Ohne konsistente Strategie, oder anders formuliert, ohne politisches Konzept dafür, was Streitkräfte wo und wie leisten sollen, ist auch der Umbau der Streitkräfte für die neuen Herausforderungen hoch problematisch. So werden kurzfristig absehbare Entwicklungen überbetont und bei der Gesamtproblematik nur noch nach Glauben und Hoffen agiert.

Diese weitgehend unbeleuchteten Problemfelder sind leicht in zwei Komplexen zu systematisieren. Da gibt es die Herausforderungen in den neuen Einsatzgebieten, in letzter Zeit also auf dem Balkan, in Asien und in Afrika. In allen Feldern ist die Bundeswehr eingesetzt worden, ohne dass strategische Ziele definiert waren und ohne dass geklärt gewesen wäre, wann und wie und unter welchen Bedingungen sie wieder herausgelöst werden würde. Außerdem sind die Kenntnisse über die Einsatzgebiete meist rudimentär, die gesellschaftlichen Verhältnisse dort weitgehend unbekannt, die Fremdheit der kulturellen Standards im Einsatzgebiet ein Problem, das sich zu einer Zeitbombe entwickeln kann, wie sich im Extremfall Irak immer mehr erweist. Die Ausbildungsdefizite, insbesondere für das Offizierkorps, in diesem Bereich sind enorm.

Das zweite große Problemfeld ist die eigene deutsche Gesellschaft und deren direkte und indirekte Einflüsse auf die Streitkräfte. Auch hier sind die Kenntnisse in der Armee unterbelichtet. Der konservative Grundzug des militärischen Selbstverständnisses lässt gesellschaftliche Entwicklungen meist als Störfaktoren erscheinen. So wird ein positiver, konstruktiver Umgang mit dem Neuen eher blockiert.

Dass sich derzeit die traditionellen Sinnstiftungsinstitutionen nicht mehr als hinreichende Orientierungsinstanzen zeigen, ist Konsequenz einer tief greifenden Veränderung des Wirtschaftsprozesses. Die deutsche Gesellschaft ist eine hoch differenzierte Dienstleistungsgesellschaft geworden, in der die neuen Tätigkeitsfelder auch neue kulturelle Standards und soziale Milieus erzeugt haben.

Problematisch für die Streitkräfte daran ist, dass wegen der traditionellen Fixierungen der Armee der Teil der Gesellschaft immer kleiner wird, aus dem sie ihren Nachwuchs rekrutieren kann. Insbesondere für die technische Modernisierung im Bereich Kommunikation und die eher unmilitärischen Aufgaben in den Einsatzgebieten braucht sie Personal, das in den Teilen der Gesellschaft verankert ist, deren Distanz zur Armee größer

7 Streitkräfte, Fähigkeiten und Technologie im 21. Jahrhundert (SFT21), Stabsstudie des Zentrums für Analysen und Studien der Bundeswehr im Auftrag des Bundesministeriums der Verteidigung, Sept. 2002. 
wird. Das geschieht nicht, weil man dort prinzipiell gegen die Streitkräfte eingestellt ist, sondern weil das Bild, das Militär von sich selber produziert, dort auf Ablehnung stößt.

Ein solches gesellschaftliches Umfeld ist nicht nur in Rekrutierungsfragen ein Problem, es ist es auch in Bezug auf die Binnenstrukturen und das Führungsverhalten in den Streitkräften. Selbst unter Soldaten mit dem beschriebenen gesellschaftlichen Konservatismus sind die neuen Wertemuster weit verbreitet. Zudem gibt es eine große Differenz zwischen den Führungseliten und den Truppenoffizieren allein schon aus Altersgründen. Denn bei den im Schnitt jüngeren Truppenoffizieren hat der Wertewandel schon weiter gegriffen als in der älteren Gruppe der Kommandeure und höheren Stabsoffiziere. So spiegelt sich die gesellschaftliche Wertedifferenzierung auch im Militär. Dagegen stehen aber die traditionellen Berufsnormen, deren intensivierte Propagierung immer mehr zu Beschwörungsformeln degeneriert.

Die Unsicherheit und Unkenntnis in diesen Fragen verleitet, in zwei Richtungen ideologisch auszuweichen. Die erste und auch häufigste ist, sich zum unpolitischen Gewaltexperten zu stilisieren, dem ein juristisch korrekt zustande gekommener militärischer Auftrag zur Legitimation reicht. Die zweite Ausweichbewegung geht in die Vergangenheit. Militär wird in Traditionszusammenhängen definiert und aus der historischen »Leistung « deutschen Militärs die Selbstverortung abgeleitet.

\subsection{Transformation der Inneren Führung?}

Diese Frage ist eindeutig zu bejahen, aber mit einer völlig anderen Stoßrichtung, als sie derzeit in Teilen der Bundeswehr diskutiert wird. Es geht vielmehr darum, das ursprüngliche Konzept, wie es sich aus den Denkschriften und Reden Baudissins ableiten lässt, wieder zur Geltung zu bringen. Innere Führung würde so zu einer Denkhilfe für alle militärischen Probleme, nicht nur für das Motivationsmanagement. Legt man dieses Analyseschema einer Analyse der derzeitigen Probleme zugrunde, lassen sich auf rationale Weise Zusammenhänge, Abhängigkeiten und Erfordernisse ableiten. Man muss nicht mehr glauben und hoffen, sondern kann analysieren und wissen, ja man kann sogar ableiten, was zu tun ist. Dann wird aber auch deutlich, dass der Soldatenberuf

- ein politischer Beruf ist,

- eines ethischen Fundamentes bedarf,

- in demokratische gesellschaftliche Verhältnisse integriert sein muss und

- politischen Zwecken und Normen unterworfen ist.

Mit diesem von Baudissin genutzten Analyseschema lassen sich auch die derzeit vielleicht heikelsten Fragen der Sicherheitspolitik hinreichend analysieren.

1. Ist es richtig, sinnvoll und Erfolg versprechend, mit Militär den Kampf gegen den Terrorismus aufzunehmen? Die innenpolitische Diskussion dazu hat ja schon vor einiger Zeit auch in Deutschland begonnen, auch wenn sie wieder eingeschlafen zu sein scheint. Wichtig aber bleibt die Frage schon deshalb, weil der wichtigste Verbündete einen solchen Krieg gegen den Terrorismus führt.
2. Was ist die entscheidende Differenz zwischen Militär der entwickelten modernen Staaten und den Gewaltorganisationen in Bürgerkriegs- und Staatsverfallsgebieten mit teilweise erheblichem kriminellem Potential, und was kann und soll modernes Militär in diesen Gebieten leisten? Oder anders gefragt, was legitimiert militärische Interventionspolitik in solchen Gebieten?

Die Beantwortung der zweiten Frage wird auch helfen, die erste zu beantworten. Was sind also die wichtigsten Merkmale und Funktionen modernen Staatenmilitärs?

Europäisches Militär wurde im 17./18. Jahrhundert erfunden, um der Brutalisierung privatisierter und entgrenzter Gewalt des Dreißigjährigen Krieges ein Ende zu setzen. Sein Zweck war Gewalteinhegung, Gewaltkontrolle und Frieden innerhalb der Staatsgrenzen und der beherrschbare Einsatz im Kriege. Dies durchzusetzen ist im Laufe der Jahrhunderte weitgehend gelungen. Die Differenzierung in Militär für den Einsatz nach außen und Polizei für den Erhalt des Friedens im Inneren ist eine kulturelle Errungenschaft Europas von größter Bedeutung.

Dieses Militär wird gekennzeichnet durch

- das legitimierte staatliche Gewaltmonopol,

- die ethisch-moralische und die juristische Legitimierung,

- die Verhältnismäßigkeit des Einsatzes der Gewaltmittel,

- den Schutz bestimmter Güter und Menschengruppen,

- völkerrechtskonformen Einsatz der Gewalt.

Diese Merkmale machen theoretisch Gewalteinhegung und Gewaltkontrolle und den rationalen, kontrollierten Einsatz wie auch die Beendigung des Gewaltgebrauchs möglich. Es sind genau diese Merkmale, die die Warlords, Bürgerkriegskämpfer, Terroristen und die kriminellen Gewalthaufen eben nicht in Anspruch nehmen. Die Regelverletzung ist ihre einzige Stärke, weil sie modernen Armeen sonst hoffnungslos unterlegen sind.

Was haben diese Ausführungen mit Innerer Führung zu tun?

1. Militärische Gewalt ist nur legitim im rechtskonformen Einsatz. Das schützt Soldaten vor unzumutbaren Forderungen der Politik oder von Vorgesetzten.

2. Rechtskonformität garantiert - zumindest theoretisch -, moralische Belastungen des Soldaten in Grenzen zu halten.

3. Der instrumentelle Einsatz von Militär lässt - ebenfalls theoretisch - die Begrenzung von Gewalt zu, kann Eskalation begrenzen, Deeskalation ermöglichen.

4. Der Zusammenhang mit der demokratischen Gesellschaft bleibt erhalten.

5. Der Soldat kann sich zumindest theoretisch darauf verlassen, nur in legitimen, militärisch verantwortbaren und politisch sinnvollen Einsätzen verwandt zu werden.

6. Der Soldat entgeht dadurch entgrenzter und brutalisierender Gewalt, die ihn, die feindliche und die eigene Gesellschaft zerrütten und traumatisieren kann.

7. Die Chance zu einer völkerrechtskonformen Auseinandersetzung wächst. 
8. Mit rechtlich eingehegter Gewalt besteht ein Instrument und die Chance, in den Bürgerkriegs- und Staatszerfallsgebieten legale und begrenzte Gewalten zu etablieren und so Nationenbildung, Staatsbildung und Frieden zu stiften.

Mit dieser Aufzählung sind indirekt auch die Leistungsgrenzen modernen Militärs umschrieben. Will Militär Militär bleiben, muss es diese Grenzen aufrechterhalten und akzeptieren. Sonst wird es zu einer genauso kriminellen Institution wie die, die es bekämpfen soll. An den Kriegsverbrechen z.B. der Wehrmacht in der Sowjetunion oder auf dem Balkan kann man die Folgen des Tabubruchs ablesen. Die Konsequenz aus dem Gesagten ist deshalb, sich ganz genau zu überlegen, ob und wie selbst eine humanitäre Intervention ihren Zweck erreichen kann. Sie wird es sicher nicht tun, wenn der Intervent im Bürgerkriegsgebiet sich dem völkerrechtswidrigen Verhalten seiner Kontrahenten anpasst.

Moderne Gesellschaften brauchen den Frieden auch außerhalb ihres Territoriums. Er ist die Voraussetzung dafür, die kulturellen Standards aufrechterhalten zu können. Dazu wurde auch die Art von Militär geschaffen, die sich an Recht und Gesetz, Disziplin und professionelle Regeln hält. Damit sind aber auch Grenzen beschrieben, über die dieses Militär nicht hinausgehen darf, weil sonst der Absturz in die Barbarei droht, selbst dann, wenn es deshalb bestimmte Aufgaben nicht erfüllen kann. Für das, was dieses Militär nicht leisten kann, müssen andere Instrumente geschärft werden, vielleicht auch einiges neu erfunden werden. Aber Militär muss Militär bleiben, darf nicht zum Sold- und Gewalthaufen degenerieren. Die sich abzeichnende Privatisierung der Gewalt durch amerikanische Sicherheitsfirmen im Irak ist aus der Sicht der europäischen Tradition und Erfahrung eindeutig der falsche Weg. Denn keiner kann die politische Loyalität solcher Privatarmeen garantieren, nicht einmal ihren Erfolg!

Was klärt dies aber in der Terrorismusfrage? Da Terroristen systematisch den Tabubruch betreiben, ihr Erfolg von der Verletzung der Rechtsordnung abhängt. Da Militär das Gleiche nicht tun darf, wenn es Militär bleiben soll, verbietet sich der Einsatz von Militär gegen Terroristen. Außerdem ist nicht ersichtlich, wie mit Truppenverbänden gegen Einzelkämpfer oder Kleingruppen vorgegangen werden kann. Militär kann also seiner eigenen Logik nach und auch nach seinen konstitutiven Wertbindungen kein geeignetes Instrument sein.

Was aber dann tun? Die theoretisch wirksamen Instrumente haben wir längst. Die Frage ist nur, ob sie auch schon faktisch hinreichend funktionieren. Es sind die Geheimdienste, der
Verfassungsschutz und die Kriminalpolizei im Vorfeld, und bei akuter Gefahr die Polizei, insbesondere über ihre Spezialformationen. Es geht also um Ausbau und Optimierung des Vorhandenen, um die Entwicklung der systematischen Zusammenarbeit, um die Aufklärung weit im Vorfeld auch im Ausland, und um die Konstruktion von Netzen, in denen sich Terroristen möglichst frühzeitig verfangen. Dazu ist sicherlich auch eine personelle und technische »Aufrüstung « dieser Institutionen nötig.

Wer glaubt, einen Krieg gegen den Terror führen zu können, oder auf einer niedrigeren Stufe der Auseinandersetzung mit militärischer Gewalt Erfolg zu haben, muss zweifach scheitern:

1. Weil Militär dazu untauglich ist, es sei denn, man verzichtet auf die rechtliche und moralisch-ethische Einbindung von Soldat und Organisation. Dann produziert man aber auch den Bruch mit den traditionellen Bindungen des Militärs. Historische Beispiele gibt es reichlich in der Militärgeschichte.

2. Weil er durch die unspezifische Art der dadurch erzwungenen Gewalteskalation

- die Wirkungen des Terrors multipliziert,

- eine Brutalisierung auch der eigenen Gesellschaft fördert,

- die Traumatisierung von Soldaten und Teilen der Gesellschaft mit unabsehbaren Folgen für die politische Psychologie riskiert,

- eine Gewöhnung an Gewalt als Mittel der Politik und zur Durchsetzung von Alltagsinteressen herbeiführt, die einen Verlust derjenigen kulturellen Standards zur Folge hat, die wiederum das Funktionieren komplexer Gesellschaften erst garantieren,

- die Legitimität des eigenen Handelns vor der Welt verliert (USA).

Aus all diesen Gründen gilt es, eine umfassende rationale Analyse im beschriebenen Sinne als Grundlage für eine tragfähige Transformation der Bundeswehr ohne Verlust an demokratischer und legitimatorischer Substanz zu entwickeln. Dazu ist mit dem hier vorgestellten ursprünglichen Konzept der Inneren Führung eine Hilfe gegeben, zu der es bis heute keine bessere Alternative gibt. Rückbesinnung ist also notwendig, aber keine auf historisierende Traditionalismen, sondern auf die Grundlagen und das ursprüngliche Konzept der Inneren Führung. Nicht sie muss transformiert werden, sondern die Armee mit ihrer Hilfe und nach ihren Prinzipien. 